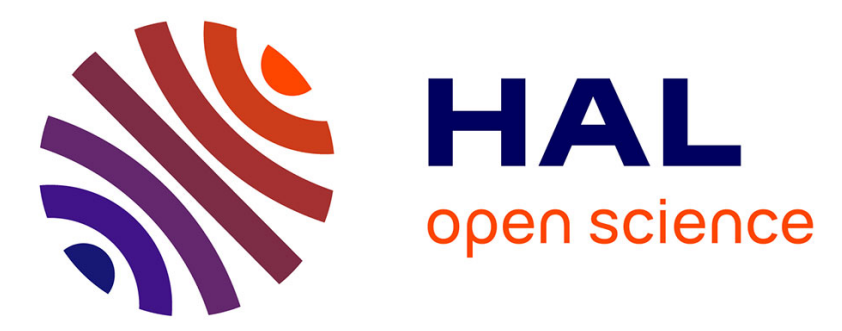

\title{
On semantic clustering and adaptive robust regression based energy-aware communication with true outliers detection in WSN
}

Srijit Chowdhury, Ambarish Roy, Abderrahim Benslimane, Chandan Giri

\section{To cite this version:}

Srijit Chowdhury, Ambarish Roy, Abderrahim Benslimane, Chandan Giri. On semantic clustering and adaptive robust regression based energy-aware communication with true outliers detection in WSN. Ad Hoc Networks, 2019, 94, pp.101934 -. 10.1016/j.adhoc.2019.101934 . hal-03487354

\section{HAL Id: hal-03487354 \\ https://hal.science/hal-03487354}

Submitted on 21 Dec 2021

HAL is a multi-disciplinary open access archive for the deposit and dissemination of scientific research documents, whether they are published or not. The documents may come from teaching and research institutions in France or abroad, or from public or private research centers.
L'archive ouverte pluridisciplinaire HAL, est destinée au dépôt et à la diffusion de documents scientifiques de niveau recherche, publiés ou non, émanant des établissements d'enseignement et de recherche français ou étrangers, des laboratoires publics ou privés.

\section{(ㄷ)(1) $\$$}

Distributed under a Creative Commons Attribution - NonCommerciall 4.0 International 
Version of Record: https://www.sciencedirect.com/science/article/pii/S1570870519303063 Manuscript_01c667dadec5e680d0dd0d3607e2beef

\title{
On Semantic Clustering and Adaptive Robust Regression Based Energy-aware Communication with True Outliers Detection in WSN
}

\author{
Srijit Chowdhury ${ }^{\mathrm{a}}$, Ambarish Roy ${ }^{\mathrm{a}}$, Abderrahim Benslimane $^{\mathrm{b}, *}$, Chandan Giri ${ }^{\mathrm{a}}$ \\ ${ }^{a}$ Department of Information Technology, Indian Institute of Engineering Science and \\ Technology, Shibpur, India \\ ${ }^{b}$ CERI/LIA University of Avignon, Avignon, France
}

\begin{abstract}
To conserve energy and enhance the lifetime of the wireless sensor network (WSN), reducing the amount of data communication by exploiting temporal and spatial correlation of sensed data is well suitable technique. So, instead of sending every data to the destination, it can be worthy of introducing a prediction method to reduce redundant data transmission by exploiting the temporal correlation of sensed data. We show that the prediction accuracy of source data depends not only on the method applied but also on the correctness of the sample data provided by the source nodes. Erroneous sample data (outliers) leads to the wrong prediction. In this paper, we propose an energy efficient SEMantic CLustering (SEMCL) model to mitigate high energy consumption problem in a clustered WSN. Our model produces energy efficient clusters by strong intra-cluster data similarity to exploit spatial correlation of data. We adopt the Robust and Efficient Weighted Least Square method (REWLS) to provide accurate data prediction with negligible errors. Because REWLS method lacks to differentiate true and false outliers and thus to improve further the Quality of Service (QoS) on data accuracy, we propose a separate algorithm, named, True Outlier Detection (TOD). Moreover, to improve the QoS in communications, a reliable backbone network based on the link quality of the data forwarding path has been implemented. Our proposed model has been simulated using real data and compared with the existing techniques to show its efficacy and superiority in terms of QoS on data accuracy, energy consumption, and network lifetime.
\end{abstract}

Keywords: Energy-aware communication; semantic clustering; prediction; robust regression; outliers, wireless sensor networks;

\footnotetext{
* Corresponding author

Email addresses: csrijitc@gmail.com (Srijit Chowdhury), ambarish268@gmail.com (Ambarish Roy), abderrahim.benslimane@univ-avignon.fr (Abderrahim Benslimane), chandangiri@gmail.com (Chandan Giri)
}

Preprint submitted to Elsevier

June 14, 2019

(C) 2019 published by Elsevier. This manuscript is made available under the CC BY NC user license https://creativecommons.org/licenses/by-nc/4.0/ 


\section{Introduction and Motivation}

One of the essential applications of the wireless sensor network (WSN) is to measure various environmental factors affecting the weather conditions. Specific weather conditions can result in partial or extensive failures of a WSN [1,2]. For example, while some environmental factors like temperature and humidity can affect the received signal strength and the link quality of a WSN adversely, a terrible weather condition like natural calamity may ultimately disrupt the working of WSN in a large geographical area. These incidents can hamper the daily routine of society that highly relies on WSNs for its various crucial activities. As the changes in weather conditions are sure to happen and the probability of incoming disaster weather is extremely time-varying, it is essential to measure the environmental factors in a regular short time interval (in seconds or minutes). It is also essential to send these measurements to the network operator or base station (BS) so that proper actions can be taken against the upcoming catastrophic weather before it strikes (for example, disaster alarm can be raised). Sending all the measurements to the BS is undesirable in an energy-constrained WSN as it introduces enormous energy consumption.

In WSN, the data communication between nodes consumes substantial energy compared to the other activities of the sensor nodes like processing or sensing data [3].

To curtail the cost of energy in data communication and to increase the operational network lifetime, reduction of redundant data in communication is required. Some studies [4] show that the weather factors (for example, temperature, light, humidity) have strong temporal correlations. Hence, a prediction method can be used at source nodes to reduce redundant data transmission by exploiting the temporal correlation of sensed data [5]. On the other hand, the nodes in WSN are densely deployed, leading to a high degree of spatial correlation among the data sensed by the neighbouring nodes [6]. The high degree of spatial correlation increases redundant data over the network, which in turn results in the consumption of a significant amount of energy in data communication. Hence, data-aware clustering method based on spatial correlation of data can be adapted to reduce redundant data transmission over the network [7].

From the discussion, it can be argued that the joint consideration of data similarity based clustering and appropriate prediction method can be useful to reduce the number of data communications which in turn can be beneficial for substantial energy savings in WSNs.

Many researchers use well-known Least Mean Square (LMS) filter based prediction algorithm (Refer to Section 2.1) as a suitable prediction method. LMS estimator minimizes mean square error (MSE) and thus produces a very low variance, i.e., the high prediction accuracy for outlier free set of observations. However, it is a well-known fact that in the presence of a single or a fraction of outliers, the estimates by LMS may vary a large from the actual observed values. Therefore, LMS is not considered as a robust estimator. In WSN, observed values of environmental variables may have outliers (inaccurate data), and thus, 
a robust estimator is required for high prediction accuracy in the presence of outliers. The robustness of an estimator can be measured in terms of breakdown point which measures the maximum fraction of outliers that may have in a given sample without spoiling the estimate completely [8]. A class of robust regression estimators has been proposed which aim to attain the maximum breakdown point and high asymptotic efficiency of estimates simultaneously. In this work, we adopt Robust and Efficient Weighted Least Square (REWLS) estimator [9] due to its outperformance over other existing robust estimators (See Section 4).

Though REWLS is a robust and efficient estimator, it lacks to differentiate between true and false outliers within a temporal dataset. It may result in inefficient prediction accuracy. For example, in the case of environmental parameters like light or wind speed, there may be a sudden change in values due to the rapid changes in the weather condition. For that reason, new observations of the environmental parameters may contain very much deviated values as compared to their previous readings. In this case, REWLS may detect these new values as outliers when compared to the previous set of observations. So, the true outlier detection is essential for high prediction accuracy, and we propose a separate algorithm for this purpose.

\subsection{Contributions}

In this work, the joint use of distributed semantic clustering and robust regression based data prediction technique has been proposed which yields a high percentage of data reduction (up to 99.5\%) in communication and enhances the operational network lifetime. The term 'semantic clustering' refers to the clustering based on data analogy. The main contributions of the proposed work can be summarised as follows:

1. An energy-efficient and distributed SEMantic CLustering (SEMCL) method has been proposed, which forms clusters with spatially correlated nodes. SEMCL adopts the REWLS method for better data accuracy of sensed values as compared to the LMS based approaches $[6,10,11]$.

2. REWLS based data prediction technique is applied to obtain high data reduction in communication and providing a high quality of service (QoS) on data accuracy. As discussed above, the REWLS method lacks to differentiate true and false outliers and thus to improve further the QoS on data accuracy, a separate algorithm, named, True Outlier Detection (TOD) has been proposed.

\subsection{Paper Organization}

The remaining parts of the paper are organized as follows: Section 2 describes the related works. Background theories related to the existing methods and the proposed method are discussed in Section 3. Section 4 demonstrates the proposed SEMCL method. The simulation results and performance analysis are described in Section 5. Finally, Section 6 concludes the work. 


\section{Related Works}

Some studies $[1,2]$ explore the effects of weather factors (e.g., temperature, humidity, etc.) in WSN and show that disastrous weather may cause severe damages to a WSN. Thus, reading the values of weather factors and forwarding these values to the BS on a regular and short time intervals is imperative, but the implementation of this task is not very straightforward in an energyconstrained network like WSN. To resolve the issue of high energy consumption in data communication and to increase the network lifetime of a WSN, a variety of clustering techniques [12] have been proposed which use various paradigms including computational intelligence(CI) [13], prediction [14] and data similarity [6]. Due to the computational complexity, CI based meta-heuristic methods are comparatively less efficient than prediction and data similarity-based approaches. Some of the existing and relevant prediction and similarity-based redundant data reduction techniques are discussed below as our proposed work considers the combination of both the prediction and the data analogy based approaches to reducing redundant data in communication.

\subsection{Prediction based approaches}

In [15], three prediction based data aggregation protocols have been proposed where a combination of Grey model and Kalman filter has been used. In this scheme, a double-queue mechanism is used to synchronize the source node and the sink. Prediction combined with Kalman Filter (PKF) [16] combines a predictor with a Kalman filter. Compared to previous works based on Kalman Filter (KF), PKF requires less computational effort while improving the reconstruction quality. Some other prediction based data aggregation protocols $[17,18]$ use autoregression and its variants. These works exploit time series data for better approximation and aggregation.

Prediction-based Data-aware Clustering (PDC) [10] exploits the temporal correlation of data and provides high prediction accuracy and low computation and communication costs. Spatial correlation of data has also been utilized in [10] to form clusters based on the similarity of data. This work does not consider the occurrence of the clustering process in every round. Instead, clusters remain unchanged until the sensor values exceed an error threshold. The main drawback of [10] is that it did not consider the residual energy of nodes in the cluster head $(\mathrm{CH})$ selection. Thus, the energy load may not be evenly distributed over the network.

In some research works, the prediction is used to reduce redundant data in communication, and the source nodes do not need to transmit every sensed data to the destination node. These prediction approaches prevent the transmission of temporally correlated data and thus help in reducing the number of transmissions in the network, which in turn minimizes the energy consumption in communication. Some recent works $[10,19]$ use dual prediction algorithm based on normalized least mean square (nLMS) method. In dual prediction method, the prediction is made at both the source node and the destination node. The prediction algorithm Optimal Step Size Least Mean Square (OSSLMS) has been 
proposed in [10], which minimizes Mean Square Deviation (MSD) to get optimal step size. Optimal step size is required for the efficient convergence of the Least Mean Square (LMS) filtering process. In another LMS based approach [11], Hierarchical least mean square (HLMS) technique has been used. In this work, two levels of hierarchical LMS filter is applied to improve the convergence speed of the LMS filter when compared with the non-hierarchical LMS techniques.

Regression method can be adapted successfully to predict the values of the parameters having a robust temporal relationship (i.e., values of such parameters change gradually over time) [20]. This statistical method has been applied widely in many real fields for its high prediction accuracy. Authors in [21] use a spatial adaptive estimation of non-parametric regression to detect the fault in autonomous systems where the fault may occur inside or outside of the systems. The work in [22] estimates the upper and lower error bounds of a real-time traffic prediction system. In [23], a regression-based model has been proposed for the prediction of health metrics, which has a significant impact on clinical practice.

\subsection{Clustering methods based on data similarity}

Data similarity based clustering has been proposed in some research works $[6$, 24]. In [24], a semantic clustering model has been proposed based on the fuzzy system. This work targets the reduction in energy consumption and improvement of data accuracy. The model exploits the spatial correlation of data to find semantic neighborhood relationships. However, this work does not use any predictive scheme and ignores the temporal correlation of data. Distributed Similarity-based Clustering and Compressed Forwarding (DSCCF) [6] constructs iso-clusters by data similarity. A dual prediction method is also applied to reduce the intra-cluster data communications. The dual prediction method uses adaptive nLMS algorithm to exploit the temporal correlation of data.

The proposed work is similar to DSCCF with the difference that instead of nLMS based prediction used in DSCCF, adaptive REWLS based prediction method has been proposed for its advantages over LMS based prediction method as discussed in Section 1. Also, a new data similarity based clustering method has been proposed which achieves superior performance as compared with the existing DSCCF clustering method in terms of formation of data similar clusters, clustering overhead and energy dissipation (See Section 5.0.2).

\section{Background theory}

\subsection{Adaptive LMS method}

Some existing prediction algorithms $[6,10,11]$ use the autoregressive model to predict time series data from some previous sample data. Autoregressive parameters are estimated by using adaptive LMS method [6]. The LMS filter accepts a sample data stream $u[t]$ of length $F_{L}$ at the time instant $t$ and calculates the prediction $v[t]$ as a linear combination of the $F_{L}$ number of previous 
samples as

$$
v[t]=w^{T}[t] u[t]
$$

where $w[t]$ is the weight vector.

The error $e[t]$ is calculated by comparing the output $v[t]$ with the desired signal $b[t]$ as

$$
e[t]=v[t]-b[t]
$$

To minimize the mean square error, the filter updates the weight vector at each time instant $t$ as

$$
w[t+1]=w[t]+\mu u[t] e[t]
$$

where $\mu$ is the step size.

After multiple rounds of prediction and weight adaptations using equations 1, 2 and 3, the output signal converges with the desired signal.

\subsection{REWLS method [9]}

The robust regression estimator REWLS simultaneously attains full efficiency and maximum breakdown point under errors having a normal distribution. In REWLS, weights are adaptively measured from a base estimator using the empirical distribution of the residuals.

Considering initial estimates of regression and scale respectively $\boldsymbol{\beta}_{0 p}$ and $\sigma_{p}$, the standardized residuals are defined as

$$
e_{k}=\frac{y_{k}-\boldsymbol{x}_{k}^{T} \boldsymbol{\beta}_{0 p}}{\sigma_{p}}
$$

where $\sigma_{p}$ is the standard deviation of $p$ samples.

If $\left|e_{k}\right|$ is large, then $\left(\boldsymbol{x}_{k}, y_{k}\right)$ is considered as an outlier. The proportion of outliers in the sample is defined as

$$
d_{p}=\max _{k>k_{0}}\left\{F^{+}\left(|e|_{(k)}\right)-\frac{(k-1)}{p}\right\}^{+}
$$

Here $\{.\}^{+}$denotes positive part, $F^{+}$is the distribution of $|X|$ where $X \sim F$, $k_{0}=\max \left\{k:|e|_{(k)}<\theta\right\}$, where $|e|_{(1)} \leq|e|_{(2)} \leq \cdots \leq|e|_{(p)}$ are the order statistics of the standardized absolute residuals and $\theta$ is some large quantile of $F^{+}$. Thus $\left\lfloor n d_{n}\right\rfloor$ observations with largest standardized absolute residuals are eliminated. The adaptive cut-off value becomes

$$
t_{p}=|e|_{\left(k_{p}\right)}
$$

where $k_{p}=p-\left\lfloor p d_{p}\right\rfloor$. It is observed that $k_{p}>k_{0}$ and $t_{p}>\theta$. Using this adaptive cut-off value the adaptive weights are measured as

$$
w_{k}= \begin{cases}1 & \text { if }\left|e_{k}\right|<t_{p} \\ 0 & \text { if }\left|e_{k}\right| \geq t_{p}\end{cases}
$$


and the REWLS estimate is

$$
\boldsymbol{\beta}_{1 p}= \begin{cases}\left(\boldsymbol{X}^{T} \boldsymbol{W} \boldsymbol{X}\right)^{-1} \boldsymbol{X}^{T} \boldsymbol{W} \boldsymbol{y} & \text { if } \sigma_{p}>0 \\ \boldsymbol{\beta}_{0 p} & \text { if } \sigma_{p}=0\end{cases}
$$

where $\boldsymbol{X}=\left(\boldsymbol{x}_{\mathbf{1}}, \ldots, \boldsymbol{x}_{\boldsymbol{p}}\right)^{T}, \boldsymbol{W}=\operatorname{diag}\left(w_{1}, \ldots, w_{p}\right)$ and $\boldsymbol{y}=\left(y_{1}, \ldots, y_{p}\right)^{T}$.

For the base estimates $\left(\boldsymbol{\beta}_{0 p}\right)$ in REWLS estimator, we use LMS estimator in our proposed prediction based communication method so that the proposed method performs better than the LMS based prediction methods especially in the presence of outliers in the sensed data.

The problem of true outlier detection using REWLS is described in the next section.

\subsubsection{Problem of REWLS estimator in detecting true outliers}

Identifying the causes and sources of outliers is essential to decide on the rejection of the detected outliers [25]. For example, if the detected outlier is a noisy data (error), it should be discarded from the sensed data and should not send to the sink to ensure high quality and accuracy of data. On the other hand, if the outlier is caused by an event (e.g., fire or chemical spills), elimination of the outlier will lead to the loss of crucial hidden information about the event. REWLS estimator is unable to distinguish the noisy data or error (we call it as the true outlier) and the outlier caused by an event (we call it as the false outlier). REWLS estimator treats all the observations which differ a large from the other similar observations in sequence as the true outlier, and thus it discards all these outliers from the future trend of the sensed data sequence (predicted data in Fig.1). In Fig. 1, the false outliers (red circled observations) are the real humidity fluctuations due to some events in nature, and thus, these observations should not be considered as errors. However, REWLS detects those points as outliers (errors).

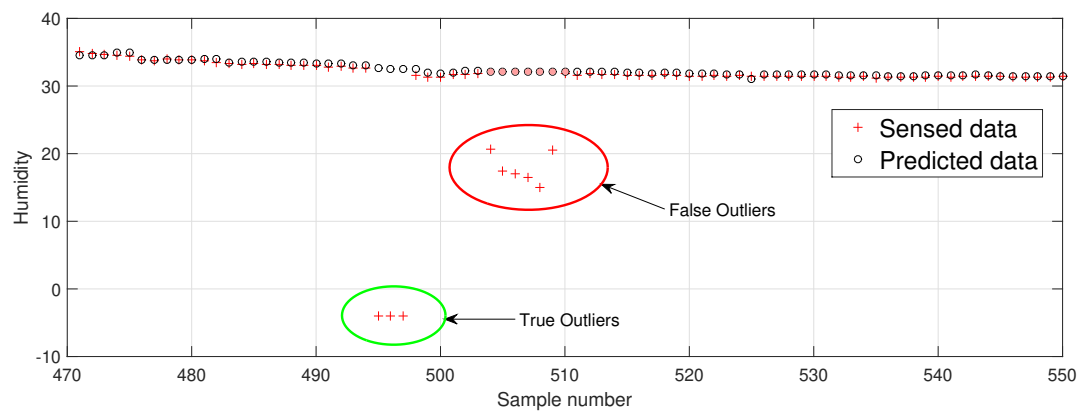

Figure 1: True outlier detection problem

The spatial correlation among observations of the neighboring nodes can help to distinguish between events and errors. Event measurements (true observations) are likely to be spatially correlated, while noisy measurements (erroneous 
observations) and sensor faults are likely to be stochastically unrelated [26]. Based on this insight, to overcome the inability of the REWLS method to detect proper outlier, a true outlier detection method named TOD has also been proposed (See Section 4.3.4).

\section{Proposed method}

\subsection{Overview}

The proposed SEMCL method consists of three phases. In the first phase, energy-efficient clusters are formed from the spatial correlation of data among the neighbour nodes. The robust regression method REWLS is used to find the spatially correlated neighbour nodes. Each non-CH node is associated with the $\mathrm{CH}$ node, which possesses high residual energy and shows high data similarity with the non-CH node. After the formation of clusters, in the second phase, a reliable backbone network structure is formed among all the $\mathrm{CH}$ nodes to communicate with the static sink. The backbone helps to choose the most energy-efficient path with strong link quality between a $\mathrm{CH}$ and the sink. In the third phase, intra-cluster and inter-cluster communication methods have been proposed for data transmission. REWLS based prediction method is employed to reduce intra-cluster data communication. Instead of receiving every sensed data from the cluster member nodes, each $\mathrm{CH}$ predicts sensed data of its associated member nodes. Then, each $\mathrm{CH}$ performs data aggregation by averaging all predicted data and sends the aggregated data to the sink through the inter-cluster backbone path. Fig. 2 describes the phases of SEMCL method.

Before describing the phases of SEMCL method, the energy model is depicted.

\subsection{Energy Model}

We use the reference of working configuration sheet of MicaZ 2.4GHz IEEE 802.15.4 motes [27] for the estimation of the expended energy in the WSN.

Energy Consumption for transmitting $k$ bytes of data is obtained as

$$
E_{t x}(k)=P_{t x} \times T_{t x}(k)
$$

where $P_{t x}=V$ olt $\times$ Ampere is the expended energy (in Joule/sec) during packet transmission and $T_{t x}$ is the transmission duration (in Seconds).

Energy Consumption for receiving $k$ bytes of data is obtained as

$$
E_{r x}(k)=P_{r x} \times T_{r x}(k)
$$

where $P_{r x}=V$ olt $\times$ Ampere is the expended energy (in Joule/sec) during packet receiving and $T_{r x}$ is the duration of receiving $k$ bytes of data.

If the data transmission rate is $R k b p s$, then the time duration of transmitting or receiving $k$ bytes of data can be calculated as $\left(\frac{k / 1000}{R}\right)$ seconds.

In the case of the clustering process, backbone formation, and data communication, various packets are transmitted over the network. A brief description of each packet is described in Table 1. 


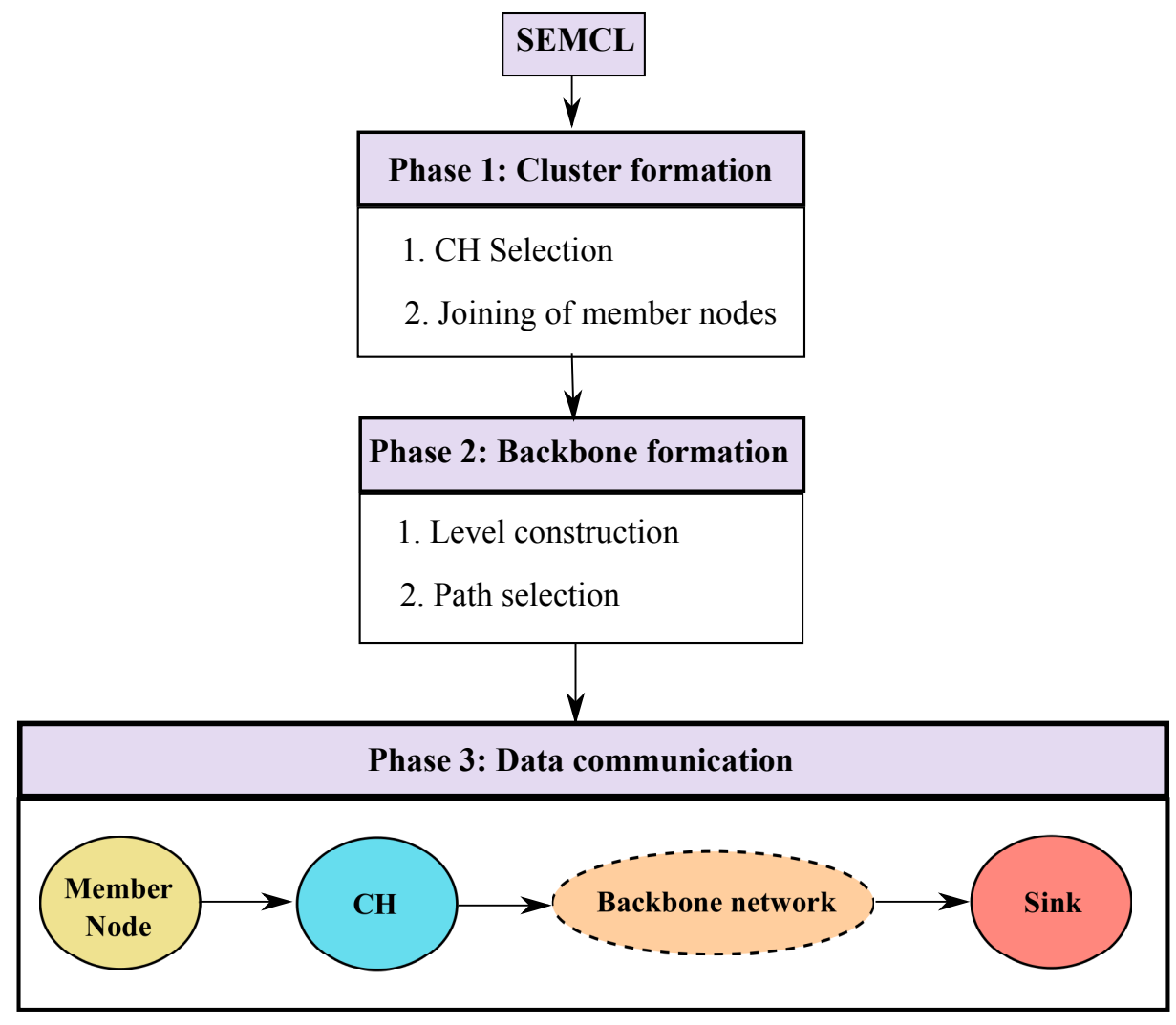

Figure 2: SEMCL method

Table 1: Packet Description

\begin{tabular}{|c|c|c|c|}
\hline $\begin{array}{c}\text { Packet } \\
\text { Name }\end{array}$ & $\begin{array}{c}\text { Packet } \\
\text { Description }\end{array}$ & $\begin{array}{c}\text { Transmission } \\
\text { Mode }\end{array}$ & Content \\
\hline$P k_{i}(1)$ & $\begin{array}{c}\text { Probable } \mathrm{CH} \\
\text { announcement } \\
\text { by node } i\end{array}$ & Broadcast & Source Id, Residual Energy, Regression Coefficients \\
\hline$P k_{i, j}(2)$ & $\begin{array}{c}\text { Packet from } \\
\text { mem node } i \text { to the } \\
\text { selected } C H_{j}\end{array}$ & Unicast & Source Id, Destination Id, Seq No, Regression Coefficients \\
\hline$P k_{i}(3)$ & $\begin{array}{c}\text { Packet from } C H_{i} \\
\text { for backbone formation }\end{array}$ & Broadcast & Source Id, Residual Energy, Backbone level \\
\hline$A c k_{i, j}$ & Packet from $C H_{i}$ to $C H_{j}$ & Unicast & Source ID, Destination Id, Ack Info \\
\hline$P k_{i, j}(4)$ & $\begin{array}{c}\text { Aggregated data Packet } \\
\text { from } C H_{i} \text { to } \\
\text { next hop node } j\end{array}$ & Unicast & Source Id, Destination Id, Aggregated Data \\
\hline
\end{tabular}




\subsection{SEMCL method}

The cluster head selection procedure of the proposed SEMCL method is described below:

\subsubsection{Cluster Head Selection}

Initially, all the nodes have non-uniform residual energy. All the nodes sense $F_{L}$ number of data from the environment and find regression coefficients. The steps are as follows:

(i) Higher energy nodes announce themselves as $\mathrm{CH}$ after waiting a specific time based on residual energy of the nodes. This specific time is measured as the ratio of a time constant $(T C)$, and Residual Energy of the announcing node say $j . T C$ is the maximum transmission time of the announcement message $P k_{j}(1)$ between two nodes.

(ii) If a node $i$ receives $P k_{j}(1)$ before it announces, then it stops announcing. Non-CH node $i$ stores all received $P k(1)$ packets in $C H_{i}^{\text {Probable }}$ list.

(iii) If a non-CH node $i$ receives a single announcement packet $P k_{j}(1)$, then it selects $j$ as its final $\mathrm{CH}$.

(iv) If a non-CH node receives multiple packets, then it performs two steps elimination to select its final $\mathrm{CH}$. In the first step, the data dissimilarity is checked. Dissimilarity value $\left(D S_{i, j}\right)$ is the Euclidean distance of regression coefficients between the announcing $C H_{j}$ and the node $i$.

If $\boldsymbol{\beta}_{i}=\left(w_{i 0}, w_{i 1}, w_{i 2}, \ldots, w_{i p}\right)$ and $\boldsymbol{\beta}_{j}=\left(w_{j 0}, w_{j 1}, w_{j 2}, \ldots, w_{j p}\right)(p$ is the number of sensed data) are the regression coefficient vectors of the nodes $\mathrm{CH}_{j}$ and $i$ respectively, then the dissimilarity value between $\boldsymbol{\beta}_{i}$ and $\boldsymbol{\beta}_{j}$ is calculated as $D S_{i, j}=\left|\left(w_{i 0}-w_{j 0}\right)+\left(w_{i 1}-w_{j 1}\right)+\left(w_{i 2}-w_{j 2}\right)+\cdots+\left(w_{i p}-w_{j p}\right)\right|$

If the dissimilarity value exceeds the predefined dissimilarity threshold (Dis_Thes), then $\mathrm{CH}_{j}$ is discarded from the $\mathrm{CH}_{i}^{\text {Probable }}$ list. In the second step, determination factor $\left(D F_{i, j}\right)$ between $C H_{j}$ and node $i$ is calculated as

$$
\begin{array}{r}
D F_{i, j}=\left(\text { Residual Energy of } C H_{j} / \text { Residual Energy of node } i\right) \\
\times\left(\text { Dis_Thes } / D S_{i, j}\right)
\end{array}
$$

(v) Node $i$ selects the $\mathrm{CH}$ that has highest $D F$ value among all the probable CHs.

(vi) Node $i$ joins the $C H_{j}$ by sending the packet $P k_{i, j}(2)$ containing node id and regression coefficients to $\mathrm{CH}_{j}$. 
(vii) After receiving a packet $P k(2)$, a $\mathrm{CH}$ stores the node-id and regression coefficients of the associated member node.

After the final selection of the $\mathrm{CHs}$, the backbone network is formed among all the selected CHs. The procedure of the backbone formation is described next.

\subsubsection{Backbone Formation}

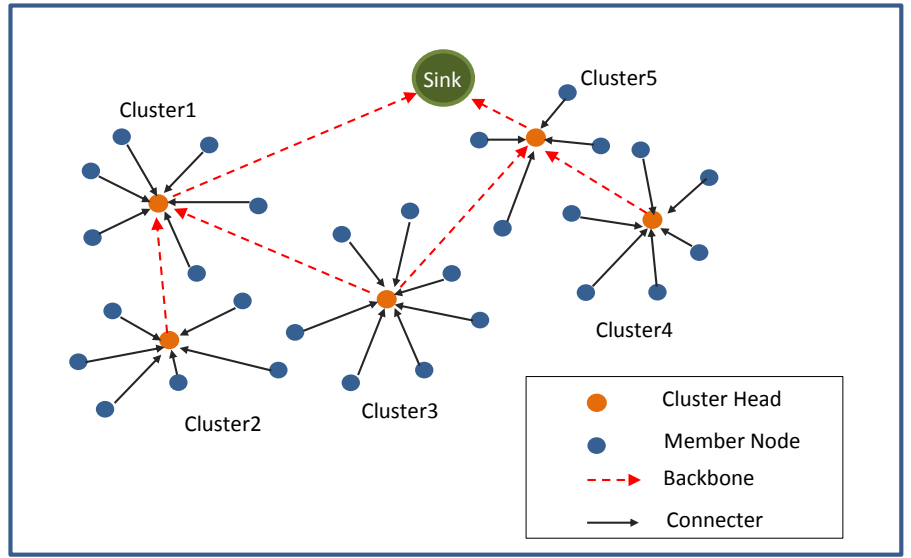

Figure 3: Distributed Clustered Network with Backbone

For inter-cluster communication, a backbone structure is formed among the selected CHs according to the quality of the link between two CHs. In this work, the quality of link $\left(\operatorname{link} Q_{i, j}\right)$ between two CHs $i$ and $j$ is measured on the basis of the link quality indicator $\left(L Q I_{i, j}\right)$ received by the destination node $j$ for the link $\{\mathrm{i}, \mathrm{j}\}$ and the residual energies of node $i$ and node $j$. The value of $L Q I_{i, j}$ is represented by a normalized parameter which varies with the inverse of the square of the distance between CHs $i$ and $j$. The quality of the link $\left(\operatorname{link} Q_{i, j}\right)$ between two nodes $i$ and $j$ is measured as

$$
\text { link } Q_{i, j}=\frac{\text { Residual energy of } i}{\text { Residual energy of } j} \times L Q I_{i, j}
$$

Each CH sets its backbone level to a very large number $(\infty)$. Backbone level of the sink is assumed to be zero. The sink starts the formation of the backbone by broadcasting the $P k(3)$ packet. If a $\mathrm{CH}$ node $j$ with level $l_{\text {evel }}$ receives a $_{j}$ single packet $P k_{i}(3)$ from the node $i$ with level level $_{i}$ where level $_{i}<$ level $_{j}$, then the $\mathrm{CH}$ node $j$ stores the value $L Q I_{i, j}$. Also, the $\mathrm{CH}$ node $j$ sets node $i$ as a predecessor (Next_Hop) and sets its new level by incrementing the level of the node $i$ by 1 . Then the $\mathrm{CH}$ node $j$ broadcasts the packet $P k_{j}(3)$ containing its new level and residual energy. If $\mathrm{CH}$ node $j$ with level level $_{j}$ receives multiple $P k(3)$ packets with the same level $\left(\right.$ level $\left._{n}\right)$ where level $_{n}<$ level $_{j}$, then the node 
$j$ calculates $\operatorname{link} Q$ value for each source $\mathrm{CH}$ node and selects the $\mathrm{CH}$ node with higher $\operatorname{link} Q$ value as its Next_Hop.

Fig. 3 describes the multi-hop relay based backbone formation among CHs. Data aggregation is done at a $\mathrm{CH}$ by averaging all predicted data of its member nodes and its sensed data. Then the $\mathrm{CH}$ sends aggregated data to the sink directly if the sink is within the range of the $\mathrm{CH}$ (see Cluster 1 and Cluster 5 in Fig. 3). Otherwise, the $\mathrm{CH}$ sends aggregated data through other $\mathrm{CH}$ in the backbone (see Cluster 2, Cluster 3, and Cluster 4 in Fig. 3). In Fig. 3, Cluster 2 and Cluster 4 forward their aggregated data packet through Cluster 1 and cluster 5, respectively, as no other alternative path is available to Cluster 2 and Cluster 4. Cluster 3 has two available paths to forward its data (through Cluster 1 or Cluster 5). Cluster 3 selects the path which possesses a higher $\operatorname{link} Q$ value at the time of data forwarding.

\subsubsection{Communication protocol between $\mathrm{CH}$ and its member nodes}

In the proposed scheme, each non- $\mathrm{CH}$ node $i$ calculates the regression coefficients $\left(\boldsymbol{\beta}_{i}\right)$ from the sample data using the REWLS method. Then each non- $\mathrm{CH}$ node sends the regression coefficients to the $\mathrm{CH}$ node. The $\mathrm{CH}$ node starts prediction for each associated non- $\mathrm{CH}$ node from the received regression coefficients.

The proposed intra cluster communication protocol is described in Algorithm 1 as follows.

Lines 3 to 6 of Algorithm 1 initialize $F_{L}$ number of sensed data $\boldsymbol{x}_{i}$ for each non-CH node $i$. Then each node $i$ generates regression coefficients $\left(\boldsymbol{\beta}_{i}\right)$ by using the REWLS method and sends those coefficients and data set $\left(\boldsymbol{\beta}_{i}, \boldsymbol{x}_{i}\right)$ to the $\mathrm{CH}$ node. Then the non-CH node $i$ starts prediction. Line numbers 7 to 21 describes the prediction state of node $i$. At a particular time instant $t(n)$, the node $i$ predicts the value $y(n)$ and also senses the value $x(n)$. Then it verifies if the error in prediction $e(n)$ exceeds the maximum tolerable error limit Emax. If $|e(n)|$ exceeds Emax then the node $i$ runs the REWLS method to adjust the regression coefficients with the help of the stored last $F_{L}$ number of data $\left(\boldsymbol{x}_{i}\right)$ including the last actual reading $x(n)$. In this way, the REWLS method is adaptively used to adjust the regression coefficients dynamically for better prediction.

On the other hand, at each time instant $t(n)$ before predicting values, the $\mathrm{CH}$ node waits for a certain amount of time to accept new regression coefficients, if any (line 30). If the $\mathrm{CH}$ node receives new coefficients and data set $\left(\boldsymbol{\beta}_{i}, \boldsymbol{x}_{i}\right)$ from any non- $\mathrm{CH}$ node $i$, it updates the corresponding coefficients temporally. Now, the $\mathrm{CH}$ checks whether the changes in coefficients are due to real changes in sensed data caused by events. Because REWLS method cannot detect the true outliers as discussed in Section 3.2.1. For this purpose, the $\mathrm{CH}$ predicts all values $y(i)$ using all changed regression coefficients (Line 33). Then it runs the TOD method (Algorithm 2) to detect true outliers. If the coefficients are changed due to the real changes in the environment, then all the spatially correlated values $y(i)$ should not possess any outlier. If $T O D$ detects any true outlier, then the corresponding $\boldsymbol{\beta}_{i}$ is discarded from the updated list of coefficients, and 


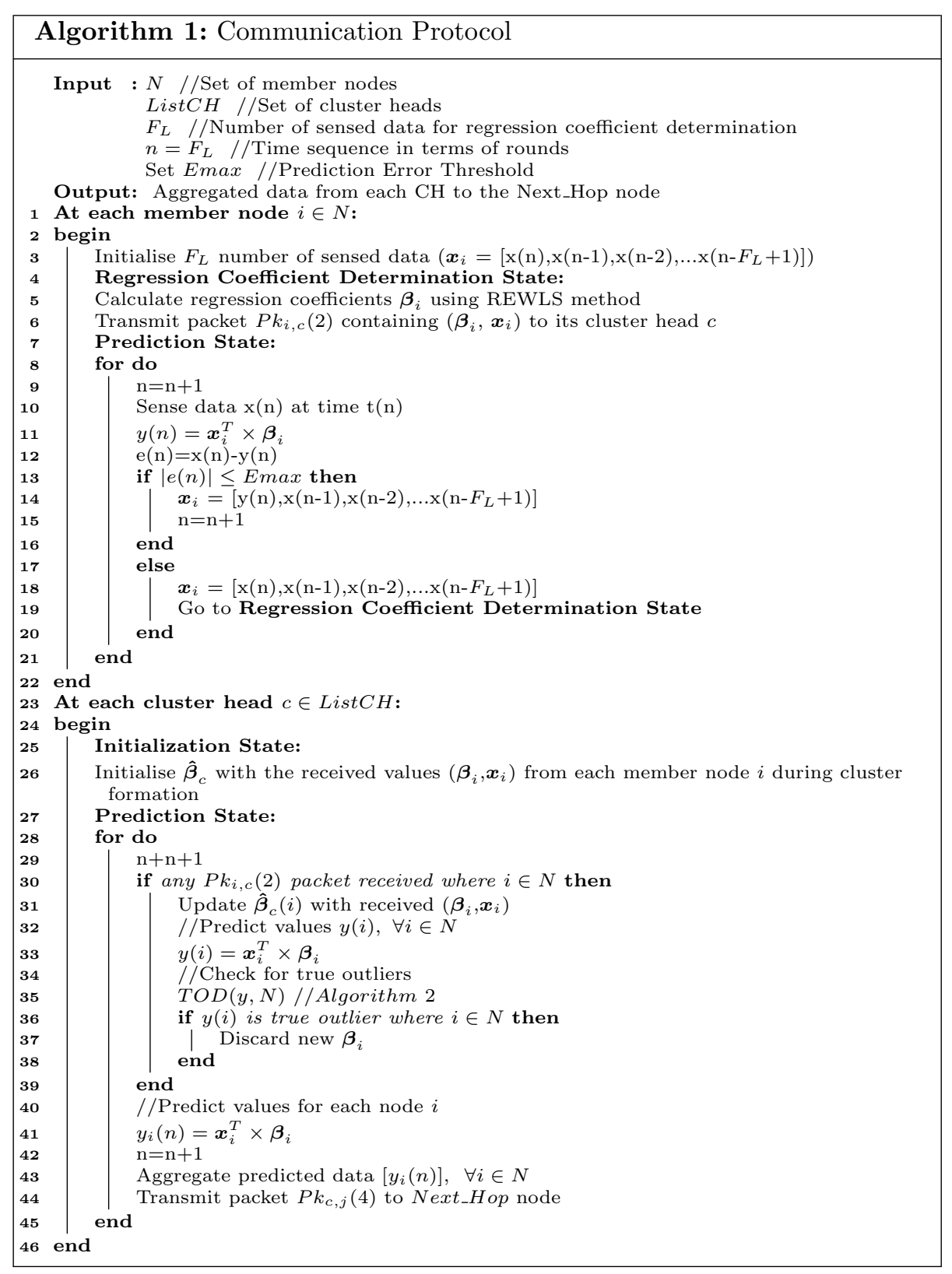


the corresponding previous regression coefficients are retained (See lines 34 to $37)$.

Now, each $\mathrm{CH}$ node predicts data for its all member nodes, aggregates all predicted data by averaging and sends to the next hop (Next_Hop) $\mathrm{CH}$ node. The Next_Hop $\mathrm{CH}$ node is determined at the backbone formation phase, as explained in Section 4.3.2.

\subsubsection{True outlier detection:}

For true outlier detection, we use interquartile range statistics [28]. The interquartile range of a dataset describes the spread of the middle fifty percent of the distribution. The interquartile range is useful to describe data sets where a few extreme values (outliers) may exist because the interquartile range is not sensitive to extreme values of the dataset. The true outlier detection method is described in Algorithm 2. The TOD algorithm first finds the median of the sorted values of the given dataset and then with the help of the median, it calculates the first and third quartiles ( $Q 1$ and $Q 3)$ [28]. Then the interquartile range $(\alpha)$ is calculated. To detect outliers, TOD calculates two thresholds $I_{1}$ and $I_{2}$ respectively over the third quartile and below the first quartile. If any value $(\boldsymbol{Y}[i])$ of the dataset lies outside of these thresholds, then it is treated as an outlier.

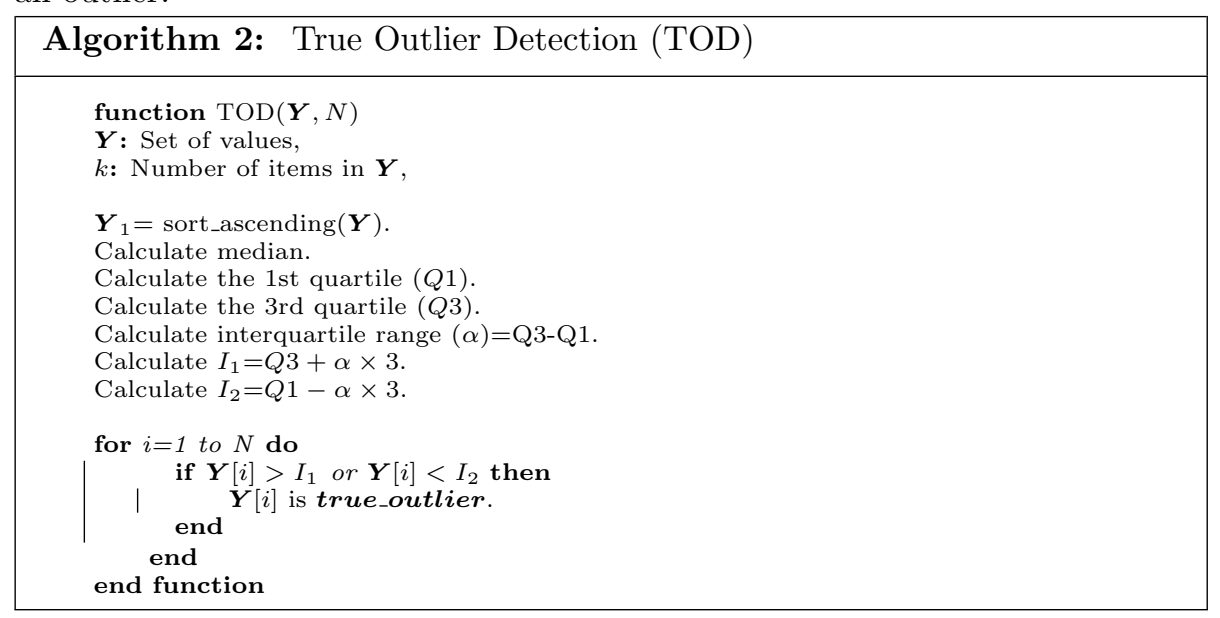

\section{Simulation results and performance analysis}

The software simulation of the proposed scheme has been performed in MATLAB [29]. We evaluate and analyze the performance of our proposed scheme for data reduction and data accuracy in transmission, data similarity in semantic clustering, overhead, energy consumption and network lifetime and compare the results with the existing works DSCCF [6], OSSLMS [10] and HLMS [11]. For simulations of the algorithms, a network which consists of spatially correlated sensor nodes is considered. For this purpose, we use publicly available real data sheet provided by the Intel Berkeley Lab [30]. Fig. 4 shows the deployment of 
the sensor nodes in the Intel Lab, where the sink is located at [0.5, 0.5]. Energy consumption of $600 \mu \mathrm{J}$ is considered for prediction [6].

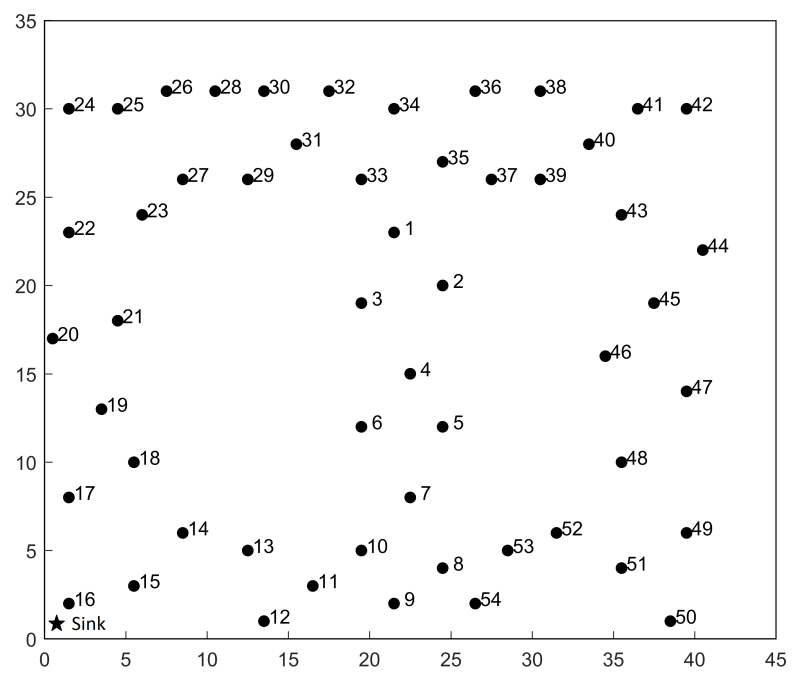

Figure 4: Sensor Node Deployment in the Intel Lab

The simulation parameters are shown in Table 2 .

Table 2: Simulation parameters

\begin{tabular}{|c|c|}
\hline Parameters & Values \\
\hline Number of nodes & 54 \\
Simulation area & $45 \mathrm{~m} \times 45 \mathrm{~m}$ \\
Transmission range & $20 \mathrm{~m}-30 \mathrm{~m}$ \\
Filter length $\left(F_{L}\right)$ & 4 \\
Emax & $0.25,0.5,0.75,1.0$ \\
Initial energy of a node $(I E)$ & $0.125 \mathrm{~J}, 0.25 \mathrm{~J}, 0.5 \mathrm{~J}, 0.75 \mathrm{~J}, 1 \mathrm{~J}$ \\
Current draw in receive mode $[27]$ & $19.7 \mathrm{~mA}$ \\
Current draw in transmit mode $[27]$ & $11 \mathrm{~mA}$ \\
Battery power (in Volt) [27] & $3 \mathrm{~V}$ \\
Data transmission rate [27] & $250 \mathrm{kbps}$ \\
Data packet size [6] & $4000 \mathrm{bits}$ \\
Control packet size [6] & $100 \mathrm{bits}$ \\
\hline
\end{tabular}

\subsubsection{Performance evaluation of REWLS based prediction}

We discuss the performance of the proposed REWLS based prediction method (In figures, we refer it as $A R E W L S$ ) and compare it with three existing LMS filter based prediction methods namely nLMS [6], OSSLMS [10] and HLMS [11]. Randomly we have selected the sensor node 20 for the evaluation. The prediction error threshold (Emax) is set to 0.25. Fig. 5(a) describes the closeness between 
the sensed data (real data) and the predicted data for 1000 sample data. The closeness is measured by the difference between the sensed data and predicted data. It can be seen in the figure that the predicted data of the REWLS based prediction method are very close to the real data when compared with other existing prediction methods. It leads to good prediction accuracy and high data reduction in communication, which is verified in Fig. 6 .

Fig. 6(a) describes the percentage of data reduction of the whole network with varying Emax. We consider 2000 samples for each node in the network. The values set for Emax are 0.25, 0.5, 0.75 and 1. From the figure, it is observed that the REWLS based prediction method yields high data reduction (97.4\% to $99.5 \%$ ) while other existing algorithms manage $86.1 \%$ to $92.5 \%$ data reduction. Thus the REWLS based prediction method has achieved $7 \%$ to $11.3 \%$ improvement in data reduction compared with the existing algorithms.

Now, we evaluate the data prediction accuracy of all algorithms by measuring the Root Mean Square Error (RMSE) [31]. RMSE measures the average deviation of the predicted values from the actual sensed data and is calculated as given in equation (11).

$$
R M S E=\sqrt{\frac{\sum_{i=1}^{n} e(n)^{2}}{t}}
$$

where $e(n)$ is the error in prediction at the $n$th time instance. It is seen from Fig. 6(b) that REWLS based prediction method yields low RMSE value in all the cases as compared with the existing algorithms. Thus REWLS based prediction method produces minimum average deviation when compared with nLMS, OSSLMS, and HLMS.

\subsubsection{Performance evaluation of SEMCL}

We evaluate the performance of the proposed clustering algorithm SEMCL and compare the results with an existing clustering algorithm DSCCF [6]. We do not consider OSSLMS [10], and HLMS [11] for this performance evaluation as those schemes have not proposed any clustering methods. We measure the clustering efficiency in terms of the average dissimilarity between a non-CH node and its associated $\mathrm{CH}$ node, clustering overhead, and energy dissipation in the clustering process.

Fig. 7 shows average dissimilarity values per round where Emax is set to 0.25 , and Dis_Thes is set to 1 . Average dissimilarity value in a round is calculated by $\frac{\sum_{i=1}^{q} D S_{i, c}}{q}$, where $D S_{i, c}$ is the dissimilarity value between a non- $\mathrm{CH}$ node $i$ and its corresponding $\mathrm{CH}$ node $c$, and $q$ is the number of non- $\mathrm{CH}$ nodes. Low dissimilarity value implies a better similarity between the non-CH node and its $\mathrm{CH}$. We observe that SEMCL possesses lower average dissimilarity than that of DSCCF.

The energy efficiency of a clustering process requires minimum overhead transmissions. In Fig. 8, we compare the overhead transmissions (in bytes) in clustering between SEMCL and DSCCF methods. From the figure, we see that SEMCL has significantly less overhead (in bytes) than that of DSCCF. Lower 


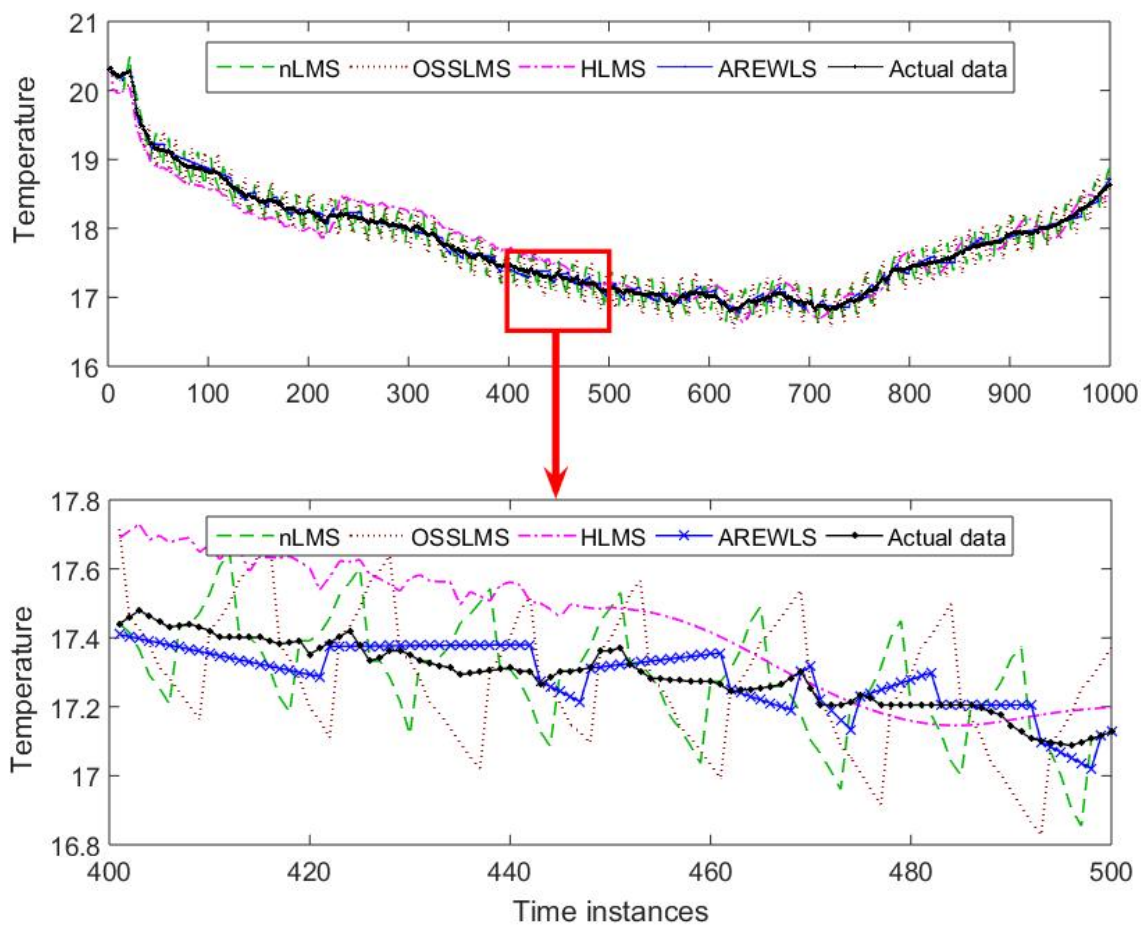

(a)
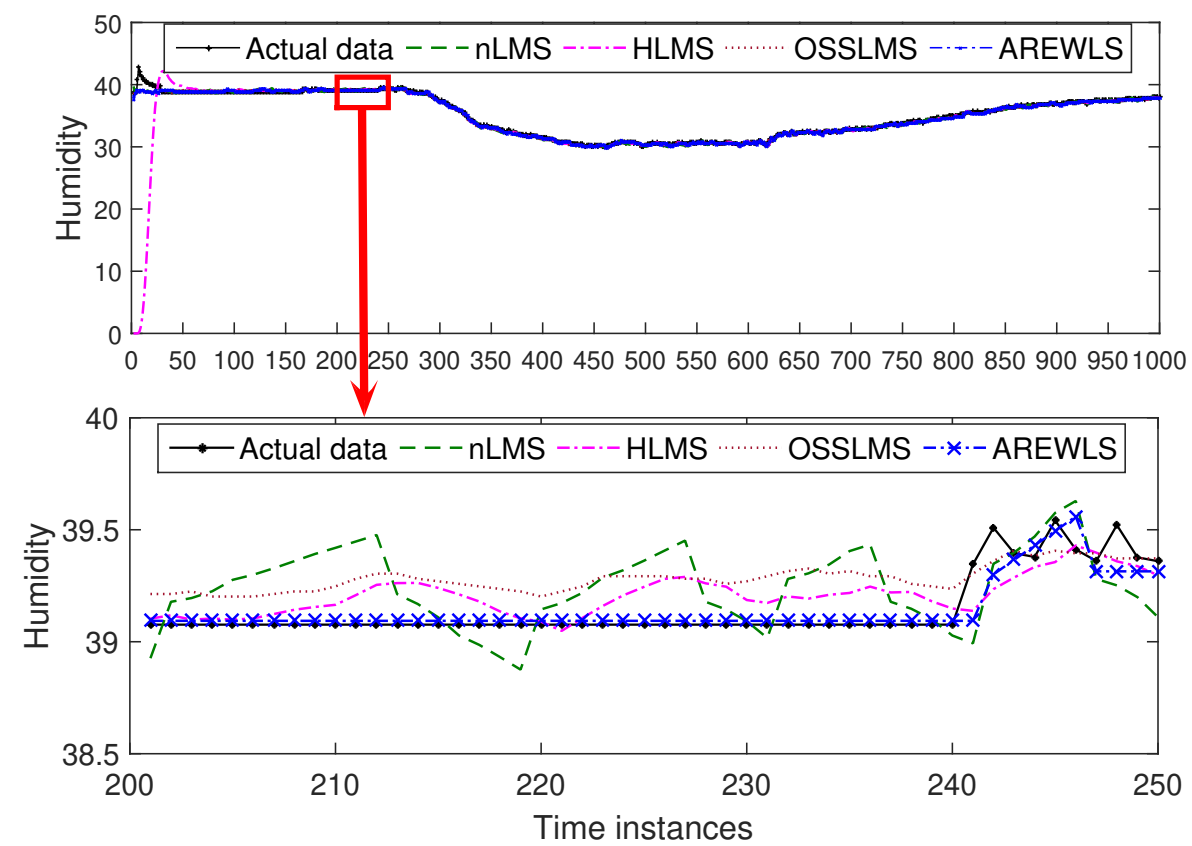

17 (b)

Figure 5: Predicted data Vs Actual data (a) Temperature (b) Humidity 


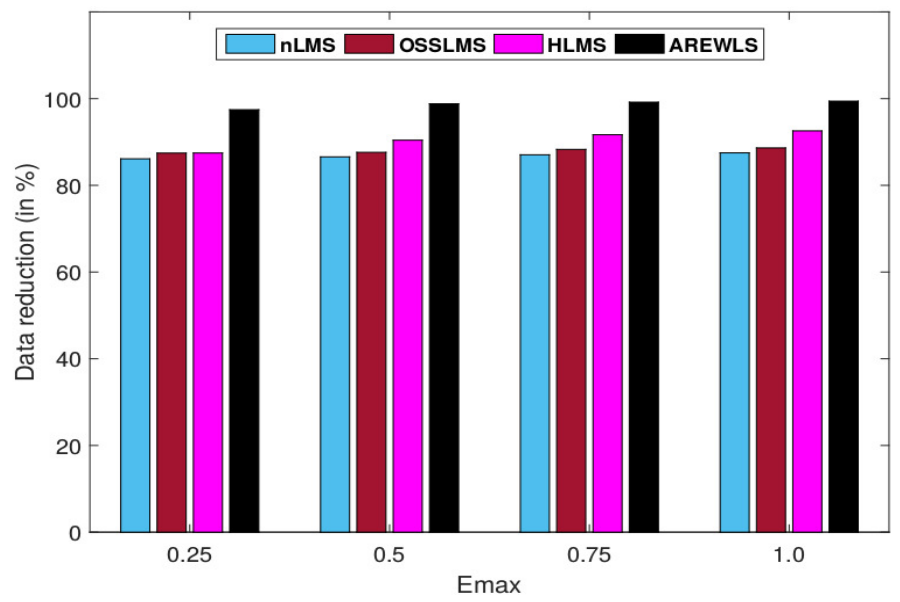

(a)

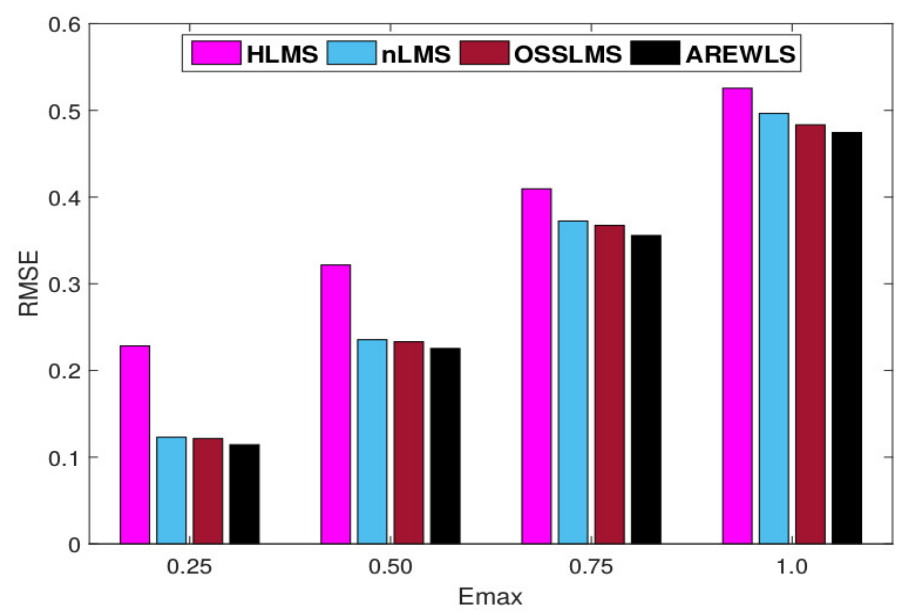

(b)

Figure 6: (a) Data reduction percentage varying with Emax (b) RMSE varying with Emax 


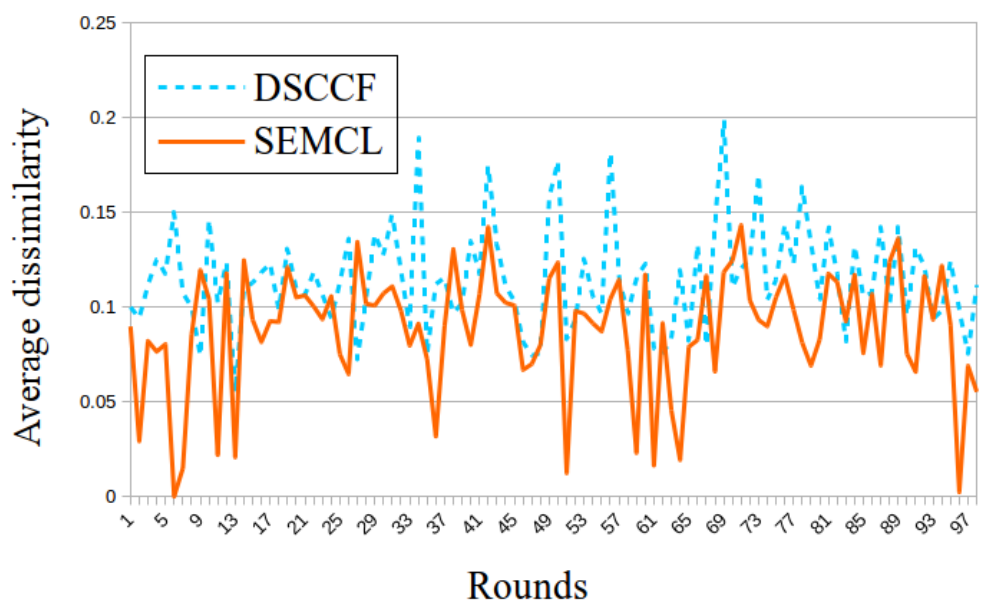

Figure 7: Average dissimilarity

overhead cost invariably leads to lower energy consumption, as shown in Fig. 9.

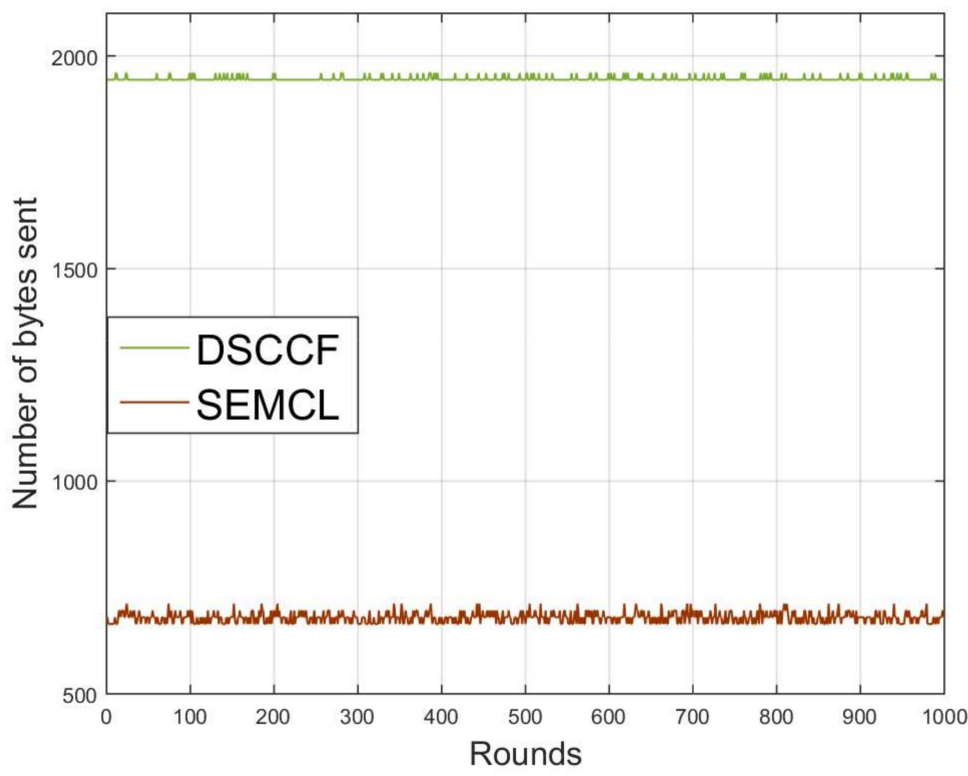

Figure 8: Clustering overhead (in bytes)

The energy consumption of the whole network per round during the cluster formation and the backbone construction is presented in Fig. 9. One can observe 
from the simulated results in both the figures that the energy consumption of SEMCL is approximately half than that of DSCCF. This less energy dissipation helps in extending the network lifetime as is depicted in figures 11 and 12.

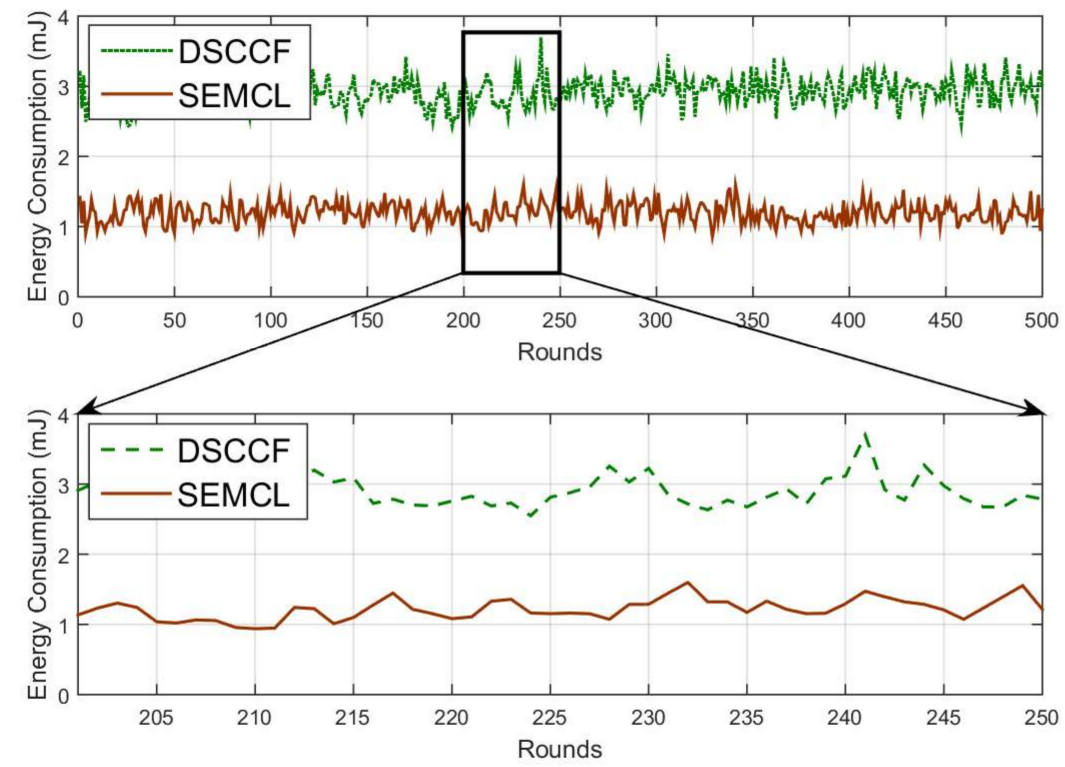

Figure 9: Energy consumption in clustering for whole network

From the simulation results, it is seen that the proposed SEMCL produces better results than DSCCF in terms of intra-cluster data similarity as well as energy consumption during cluster formation. In the next section, we evaluate the performance of the overall system.

\subsubsection{Performance evaluation of the overall system}

We evaluate the overall system performance of our proposed algorithm in terms of scalability, energy consumption, and network lifetime. Fig. 10 shows the cumulative network energy consumption per round. In the experiment, we set $I E$ to $0.125 \mathrm{~J}$ and Dis_Thes to 2. From the figure, it can be seen that the SEMCL achieves per round energy dissipation lower than that of the DSCCF.

Figures 11 and 12 compare the proposed method SEMCL with DSCCF for the network lifetime in terms of first node death, half node death and last node death respectively. To prove the scalability of the proposed model, we evaluate the network lifetime varying with $I E$ and Emax in figures 11 and 12 respectively. In all the cases of first, half and last node death, SEMCL runs for more number of rounds than DSCCF. We see that the number of rounds increases with the increase in Emax (Fig. 11); because as the upper bound of the error threshold increases, the algorithm has the scope to make a correct prediction within a wider error range. Thus, the number of successful predictions is increased, 


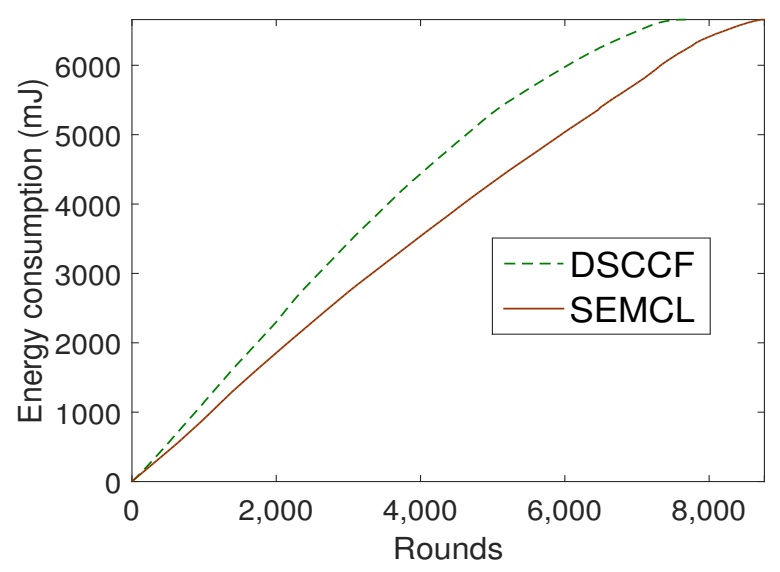

Figure 10: Network energy consumption per round

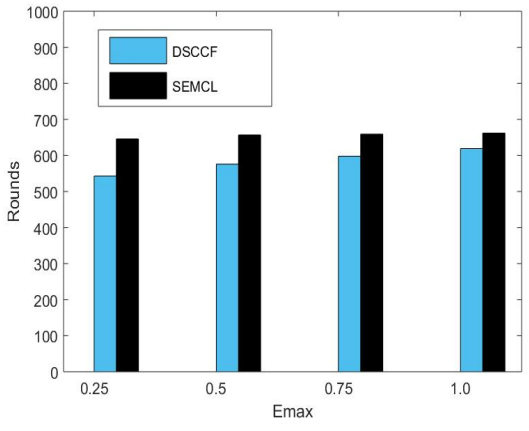

(a)

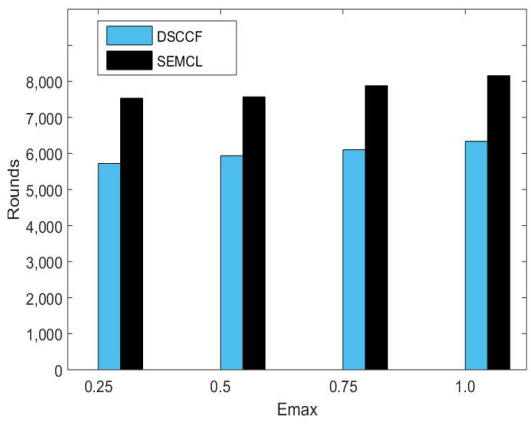

(b)

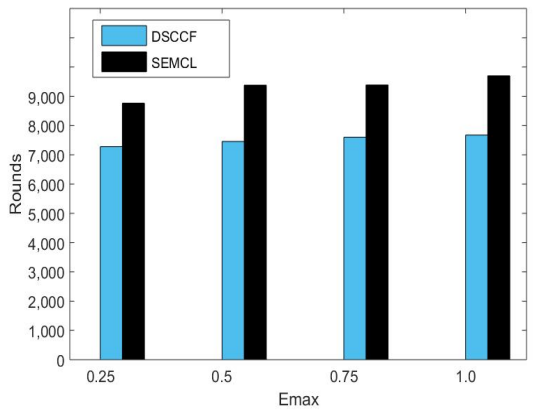

(c)

Figure 11: Node death varying with Emax (a) First Node Death (b) Half Node Death (c) Last Node Death 


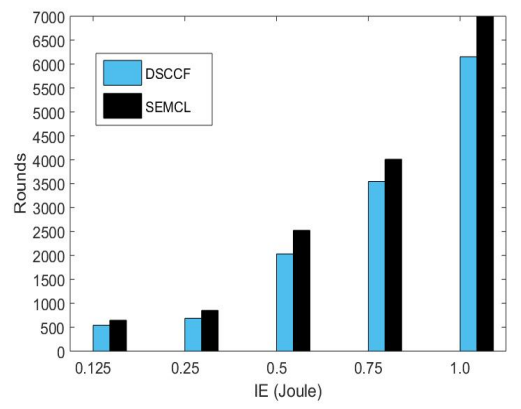

(a)

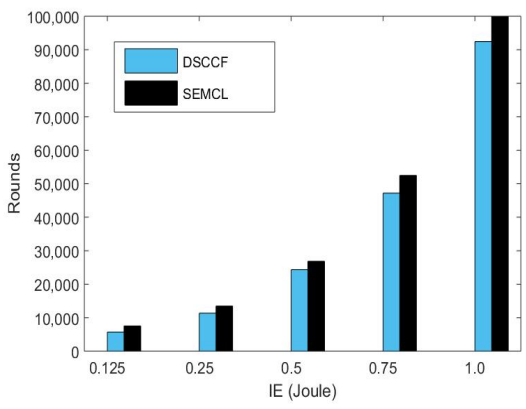

(b)

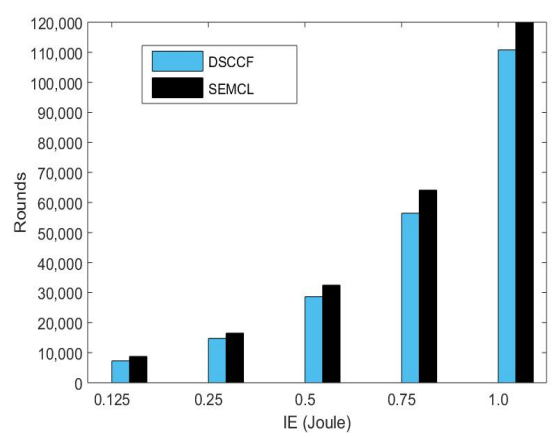

(c)

Figure 12: Node death varying with Initial energy(IE) (a) First Node Death (b) Half Node Death (c) Last Node Death 
which in effect reduces the number of data communications of individual nodes. It is also seen that in the different scenarios with varying $I E$ (Fig. 12), SEMCL outperforms DSCCF in terms of network lifetime. The overall percentage of improved network lifetime of SEMCL over DSCCF is $4 \%$ to $36 \%$.

\section{Conclusion}

This work proposes a semantic clustering method (SEMCL) which exploits temporal and spatial correlations of data to form an efficient data collection framework in a sensor network. A communication protocol is also proposed for intra-cluster correspondence, which utilizes the REWLS based prediction method. Experimental results show that the REWLS based prediction method achieves high accuracy on data prediction (up to 99.5\%); this greatly helps in reduction of data in intra-cluster data communication which in turn saves network energy to a great extent. This work also depicts an efficient method of inter-cluster communications by forming an LQI based backbone structure. The proposed work is compared with the existing works in various network settings. Through rigorous simulation, it is shown that the proposed work showcases better results in terms of QoS on data accuracy and reliability, data reduction, energy consumption, and network lifetime.

\section{References}

[1] J. Luomala and I. Hakala. Effects of temperature and humidity on radio signal strength in outdoor wireless sensor networks. In 2015 Federated Conference on Computer Science and Information Systems (FedCSIS), pages 1247-1255, Sept 2015.

[2] M. Tornatore, J. Andr, P. Babarczi, T. Braun, E. Flstad, P. Heegaard, A. Hmaity, M. Furdek, L. Jorge, W. Kmiecik, C. Mas Machuca, L. Martins, C. Medeiros, F. Musumeci, A. Pai, J. Rak, S. Simpson, R. Travanca, and A. Voyiatzis. A survey on network resiliency methodologies against weather-based disruptions. In 2016 8th International Workshop on Resilient Networks Design and Modeling (RNDM), pages 23-34, Sept 2016.

[3] I.F. Akyildiz, W. Su, Y. Sankarasubramaniam, and E. Cayirci. Wireless sensor networks: a survey. Computer Networks, 38(4):393 - 422, 2002.

[4] F. Wang, S. Wu, K. Wang, and X. Hu. Energy-efficient clustering using correlation and random update based on data change rate for wireless sensor networks. IEEE Sensors Journal, 16(13):5471-5480, July 2016.

[5] Adwitiya Sinha and DK Lobiyal. Prediction models for energy efficient data aggregation in wireless sensor network. Wireless Personal Communications, 84(2):1325-1343, 2015. 
[6] Muruganantham Arunraja, Veluchamy Malathi, and Erulappan Sakthivel. Distributed similarity based clustering and compressed forwarding for wireless sensor networks. ISA transactions, 59:180-192, 2015.

[7] Apoorva Jindal and Konstantinos Psounis. Modeling spatially correlated data in sensor networks. ACM Transactions on Sensor Networks (TOSN), 2(4):466-499, 2006.

[8] Victor J. Yohai. High breakdown-point and high efficiency robust estimates for regression. Ann. Statist., 15(2):642-656, 061987.

[9] Daniel Gervini and Victor J. Yohai. A class of robust and fully efficient regression estimators. Ann. Statist., 30(2):583-616, 042002.

[10] Mou Wu, Liansheng Tan, and Naixue Xiong. Data prediction, compression, and recovery in clustered wireless sensor networks for environmental monitoring applications. Information Sciences, 329:800-818, 2016.

[11] Liansheng Tan and Mou Wu. Data reduction in wireless sensor networks: A hierarchical LMS prediction approach. IEEE Sensors Journal, 16(6):1708$1715,2016$.

[12] M. Mehdi Afsar and Mohammad-H. Tayarani-N. Clustering in sensor networks: A literature survey. Journal of Network and Computer Applications, 46:198 - 226, 2014.

[13] Palvinder Singh Mann and Satvir Singh. Energy efficient clustering protocol based on improved metaheuristic in wireless sensor networks. Journal of Network and Computer Applications, 83:40 - 52, 2017.

[14] Majid Ashouri, Hamed Yousefi, Javad Basiri, Ali Mohammad Afshin Hemmatyar, and Ali Movaghar. Pdc: Prediction-based data-aware clustering in wireless sensor networks. Journal of Parallel and Distributed Computing, 81:24-35, 2015.

[15] Guiyi Wei, Yun Ling, Binfeng Guo, Bin Xiao, and Athanasios V Vasilakos. Prediction-based data aggregation in wireless sensor networks: Combining grey model and kalman filter. Computer Communications, 34(6):793-802, 2011.

[16] Yanqiu Huang, Wanli Yu, Christof Osewold, and Alberto Garcia-Ortiz. Analysis of pkf: A communication cost reduction scheme for wireless sensor networks. IEEE Transactions on Wireless Communications, 15(2):843-856, 2016.

[17] Suat Ozdemir and Yang Xiao. Polynomial regression based secure data aggregation for wireless sensor networks. In Global Telecommunications Conference (GLOBECOM 2011), 2011 IEEE, pages 1-5. IEEE, 2011. 
[18] Carlos Carvalho, Danielo G Gomes, Nazim Agoulmine, and José Neuman de Souza. Improving prediction accuracy for wsn data reduction by applying multivariate spatio-temporal correlation. Sensors, 11(11):10010-10037, 2011.

[19] Hongbo Jiang, Shudong Jin, and Chonggang Wang. Prediction or not? an energy-efficient framework for clustering-based data collection in wireless sensor networks. IEEE Transactions on Parallel and Distributed Systems, 22(6):1064-1071, 2011.

[20] H. Firouzi, A. O. Hero, and B. Rajaratnam. Two-stage sampling, prediction and adaptive regression via correlation screening. IEEE Transactions on Information Theory, 63(1):698-714, Jan 2017.

[21] Alexander Kalmuk, Oleg Granichin, Olga Granichina, and Mingyue Ding. A dynamic threshold based algorithm for change detection in autonomous systems. IFAC-PapersOnLine, 49(13):141 - 145, 2016. 12th IFAC Workshop on Adaptation and Learning in Control and Signal Processing ALCOSP 2016.

[22] F. C. Pereira, C. Antoniou, J. A. Fargas, and M. Ben-Akiva. A metamodel for estimating error bounds in real-time traffic prediction systems. IEEE Transactions on Intelligent Transportation Systems, 15(3):1310-1322, June 2014.

[23] S. Robben, G. Englebienne, and B. Krse. Delta features from ambient sensor data are good predictors of change in functional health. IEEE Journal of Biomedical and Health Informatics, 21(4):986-993, July 2017.

[24] Atslands R. Rocha, Luci Pirmez, Flvia C. Delicato, rico Lemos, Igor Santos, Danielo G. Gomes, and Jos Neuman de Souza. \{WSNs\} clustering based on semantic neighborhood relationships. Computer Networks, 56(5):1627 $1645,2012$.

[25] M. Bahrepour, Y. Zhang, N. Meratnia, and P. J. M. Havinga. Use of event detection approaches for outlier detection in wireless sensor networks. In 2009 International Conference on Intelligent Sensors, Sensor Networks and Information Processing (ISSNIP), pages 439-444, Dec 2009.

[26] X. Luo, M. Dong, and Y. Huang. On distributed fault-tolerant detection in wireless sensor networks. IEEE Transactions on Computers, 55(1):58-70, Jan 2006.

[27] Micaz. http://www.memsic.com/userfiles/files/Datasheets/WSN/ micaz_datasheet-t.pdf.

[28] Graham Upton and Ian Cook. Understanding Statistics. Oxford University Press, 1996.

[29] Matlab. http://in.mathworks.com/help/matlab/. 
[30] Intel lab data, 2004. http://db.csail.mit.edu/labdata/labdata.html.

[31] Lu Zhu, Zhiqun Huang, Yuanyuan Liu, Chaozheng Yue, and Baishan $\mathrm{Ci}$. The nonparametric bayesian dictionary learning based interpolation method for wsns missing data. AEU - International Journal of Electronics and Communications, 79:267 - 274, 2017. 